\title{
Abstracts Read at the Scientific Meeting of the II Euro-Mediterranean and Ibero-American Congress of Paraplegia
}

Held at Toledo, Spain in April, 1985

\section{Spinal cord compression with malignant lymphoma \\ Ximena Maldonado, M.D.; Jorge Salazar M.D.; Jesus Vaquero, M.D. \\ Departments of Haematology and Neurosurgery, Puerto de Hierro Clinic Autonomous University, Madrid, Spain.}

The treatment of spinal cord compression by lymphoma remains a topic of interest as about $6^{\circ}{ }_{0}$ of the patients with lymphoma present with this complication. We present our experience with seven cases treated with both surgical decompression and radiotherapy. Out of 39 patients with spinal metastases about $18^{\circ}{ }_{0}$ have a malignant lymphoma. We studied six females and one male with a mean age of 21 years. Fifty-seven percent showed a syndrome of a complete spinal cord lesion and $42 \cdot 8^{\circ}$. incomplete involvement. Eighty-five percent had radicular pain, $71^{\circ}$ o parasthesiae, $87 \cdot 5^{\circ}{ }_{0}$ weakness and gait disturbance and $71.4^{\circ}{ }_{0}$ sphincter dysfunction. Paraplegia was present in two cases. The dorsal region was the most frequently affected in five cases. The evolution of spinal cord compression was acute (less than 24 hours), in two cases and progressive in five cases. Simple X-ray examination showed lytic and blastic bony alterations in $28.5 \%$ of cases. Bone scan studies were abnormal in three cases $(42.8 \%)$. Myelography showed an epidural space-occupying lesion in all cases, five with a complete block and two with incomplete. Laminectomy was performed before radiotherapy in two cases with severe neurologic deficit with rapid progression of their manifestations. Although the mean survival was only 6 months after the spinal cord compression was treated, $71^{\circ}$, showed improvement in pain discomfort, $42 \cdot 8^{\circ}{ }_{0}$ recovered motor function and walked unaided, including one patient with previous paraplegia. The indications and controversial use of surgical or conservative treatment are discussed. 


\section{Scott prothesis in urinary incontinence: Case report}

J. A. Blas Quilez, M.D., Senior Consultant Doctor in Urology and J. Fuentes Garcia, M.D., Service Chief in Urology.

Hospital Nacional de Parplejicos, Toledo, Spain.

A female patient, 10 years of age, with paraplegia caused by a lumbosacral myelomeningocoele, is described.

This patient had constant urinary incontinence which made social activities impossible (school, parties etc.). She was unable to go anywhere because she also had a pronounced psychological complex.

We evaluated the social, intellectual and psychological environment of this patient and decided to insert an artificial urinary sphincter around the bladder neck: a Scott prothesis.

To avoid bladder neck necrosis that could be caused by ischaemia, we calibrated the Scott prothesis with a very low pressure, so that she can maintain continency for approximately 3 or 4 hours. After this time some urine will escape. We instructed the patient to release the pressure on the sphincter every 3 or 4 hours.

We have examined the patient after 1 year and she continues with the same degree of continence. No complications arose in the use of the Scott prothesis.

We feel that this is a good method to deal with urinary incontinence, although patients must be selected with great care. Intellect, social environment and ability to manipulate the prothesis are important, and the urinary system must be normal. It is contra-indicated in tetraplegic patients, previous surgery in the implant area, a small and fibrotic bladder or if there is degenerative disease.

\section{Orthesis in spinal cord injuries}

V. Forner, M.D.D.Phy.Med.; M. Sotos; J. Navarro; E. Perez Martinez Department of Rehabilitation, 'La Fe' Hospital, Valencia, Spain.

Patients with spinal cord injuries require different types of orthesis in the acute as well as in the late stages of the lesion to prevent deformities in the upper and lower limbs and to increase function.

Attention has been focussed on the prescription of lower limb ortheses to allow walking with crutches as there are different criteria regarding the importance of the gait as the main goal in the rehabilitation process.

We have reviewed 66 complete and 111 incomplete patients treated at ' $\mathrm{La} \mathrm{Fe}$ ' Hospital in Valencia.

Males were younger and acquired a higher degree of independence for A.D.L. than females, probably due to the higher incidence of traumatic aetiology in the former.

We had prescribed long braces to $63 \cdot 6^{\circ}$ of the complete patients but only $31.6 \%$ of them were using them for standing and for walking exercises at the time of the interview.

Only two young patients with thoracic injuries preferred walking with braces and crutches to wheelchair locomotion. Short braces were prescribed for $4.5^{\circ} \%$ of the patients with complete lower lesions and all of them were using braces for walking. Therefore only $12.4^{\circ}$ o of patients with complete lesions used the long braces for standing exercise and walking. Community walking is exceptional in 
complete patients, at least in our country.

Walking with crutches and braces is not a realistic goal for the majority of patients and they should not be disappointed if they do not achieve that at the end of the rehabilitation process.

The hospitalisation should not be prolonged in order to achieve something which is only attained by young, healthy and well motivated patients; otherwise the rehabilitation process may be expensive and even frustrating. 